\title{
Ionization Structure and Mass Loss for Rapidly-Rotating Near Main-Sequence B Stars
}

\author{
J.E. Bjorkman and B.P. Abbott \\ Ritter Observatory, Dept. of Physics \& Astronomy, \\ University of Toledo, Toledo, $\mathrm{OH}$ 43606-3390, USA
}

\begin{abstract}
Using the wind-compressed disk model to determine the density and velocity of a rapidly rotating wind, we calculate the 2-D ionization structure and corresponding line profiles. We find that previous estimates of the mass-loss rate based on spherically symmetric models may be a factor of 5-10 too small.
\end{abstract}

\section{Introduction}

Rapid rotation can produce large equatorial density enhancements in the stellar wind, leading to a corresponding latitudinal ionization gradient. The resulting 2-D ionization structure can significantly alter the UV resonance line profiles, which are used to measure the mass-loss rate and wind terminal speed.

\section{Model}

To determine the ionization state of the wind, we modified a spherically symmetric NLTE ionization code (MacFarlane et al. 1993) to construct a 2-D piecewise spherical model of the ionization fractions of $\mathrm{H}, \mathrm{He}, \mathrm{C}, \mathrm{N}, \mathrm{O}$, and $\mathrm{Si}$. In the ionization model, we use the density and velocity obtained from the windcompressed disk model (Bjorkman \& Cassinelli 1993). With these ionization fractions, we then calculate synthetic line profiles using a three-dimensional Monte Carlo simulation of the radiation transport for resonance line scattering. The particular UV resonance lines we calculate are: Si III, Si IV, C III, C IV, and $\mathrm{N} v$.

For an example case, we employ a hypothetical B2.5IV star, since this is where rotational effects are strongest, the envelope is becoming optically thin to the X-rays, and the mass-loss rates are especially uncertain. For our baseline mass-loss rate we choose $\dot{M}=10^{-10} \mathrm{M}_{\odot} \mathrm{yr}^{-1}$ (Snow 1981; Prinja 1989). Since MacFarlane, Cohen, \& Wang (1994) have shown that X-rays dominate the photoionization of $\mathrm{B}$ star winds, we also choose a baseline $\mathrm{X}$-ray emission measure, $\log E M_{\mathrm{X}}=53.0 \mathrm{~cm}^{-3}$, based on ROSAT observations of B stars (Cohen, Cassinelli, \& MacFarlane 1997). The X-rays are assumed to be uniformly distributed in the wind. We then varied the mass-loss rate and X-ray emission measure to determine the allowable range of mass-loss rates and X-ray emission measures that would produce acceptable line profiles. 
(a)

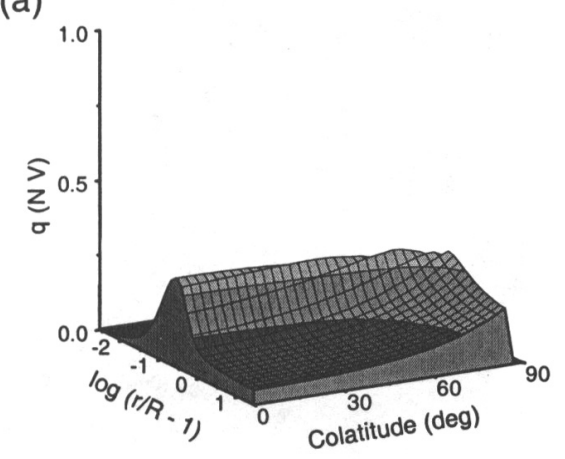

(b)

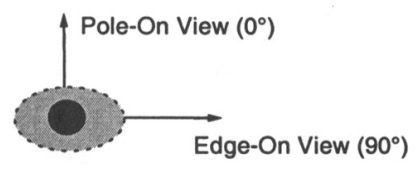

(c)

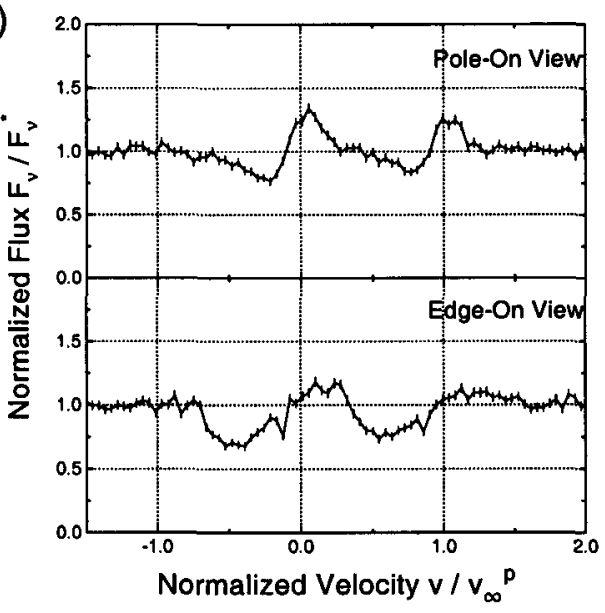

Figure 1. Ionization fraction and line profiles for $\mathrm{N} \mathrm{v}$. Shown in panel (a) is the ionization fraction, which is higher in the equatorial region and lower in the polar regions (at large $r$ ). Panel (b) illustrates the iso-ionization contours, showing two viewing angles. The line profiles (c) for each of these viewing angles show significant differences between the emission and absorption equivalent widths. For the pole-on view, the emission is larger than the absorption, while for the edge-on view, absorption is larger than emission. Thus the ratio of the emission to absorption equivalent widths could be an indicator of viewing angle, if the dominant ionization state is known. 

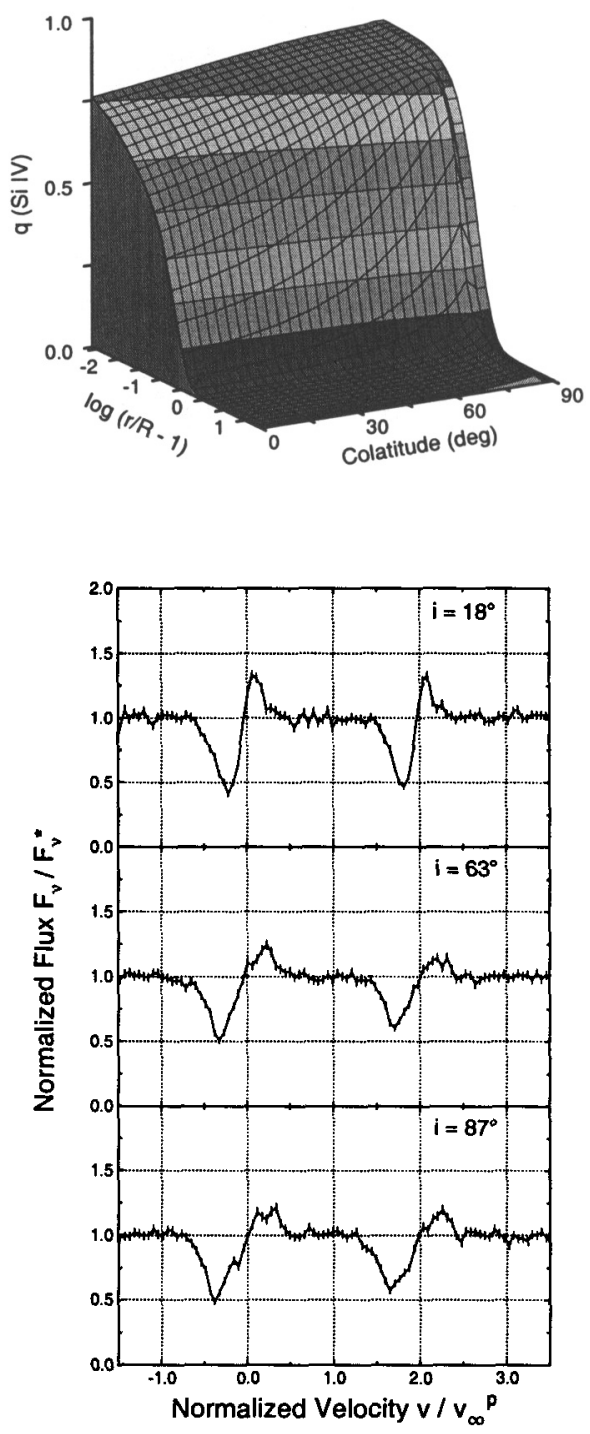

Figure 2. Ionization fraction and line profiles for Si IV. The lack of emission is a result of three phenomena: 1) the ion only exists close to the star; 2) the ion is not optically thick as $r \rightarrow \infty$; and 3) occultation effects remove the red-shifted emission. Note that although the terminal speed decreases from pole to equator, the edge velocity actually increases in this case. Near the equator, Si IV exists at larger radii than at the pole, allowing absorption at higher velocity despite the lower terminal speed. This anti-correlation demonstrates that $v_{\text {edge }}$ is not always a reliable measure of the terminal speed of the wind and can produce misleading results when looking for trends of wind properties with inclination angle. 


\section{Results}

Figures 1 and 2 show the ionization fractions and line profiles for two interesting cases. The approximate scaling for the ionization fraction is

$$
q_{i} \approx q_{i_{0}}\left(\frac{W_{X}}{n_{e} Q_{i}}\right)^{\Delta i},
$$

where $q_{i_{0}}$ is the ionization fraction of the dominant state, $W_{X}$ is the X-ray dilution factor, $n_{e}$ is the electron density, $Q_{i}$ is a constant which depends on the ratio of the recombination and photoionization rates, and $\Delta i=i-i_{0}$ is the number of ionization stages away from the dominant ionization state, $i_{0}$. For ions above the dominant ionization state $(\Delta i>0)$, the ion is enhanced in the polar regions and depleted in the equatorial regions. For ions below the dominant state, the ion is increased near the equator and destroyed near the poles. This behavior produces either excess absorption or excess emission depending on viewing angle (see Fig. 1). It can also influence the edge velocity, making the edge velocity a poor indicator of the terminal speed (see Fig. 2).

\section{Conclusions}

For the B2.5IV star chosen for this study, we find the mass-loss rate to be a few $10^{-9} \mathrm{M}_{\odot} \mathrm{yr}^{-1}$ and the $\mathrm{X}$-ray emission measure $\log E M_{\mathrm{X}} \sim 52.5 \mathrm{~cm}^{-3}$. This result is consistent with the observed $\mathrm{X}$-ray luminosities as well as typical $\mathrm{UV}$ resonance line profiles. Our mass-loss rate is a factor of 5-10 higher than that determined from previous spherically symmetric models.

We also examined the effects of the latitudinal density gradient on the line profiles. We find that the line profiles are sensitive to two-dimensional effects, showing disproportionate amounts of emission versus absorption as the viewing location changes from pole to equator. For example, ions below the dominant ionization state are more abundant in the equatorial regions and depleted near the pole. These ions show enhanced absorption for an edge-on observer, while a pole-on observer sees enhanced emission. For ions above the dominant ionization stage, the situation is reversed. Finally, we find in some instances that despite the fact that the wind terminal speed decreases toward the equator, the blue edge velocity can actually increase with increasing inclination angle.

Acknowledgments. This work has been supported under NASA grants NAG5-3447 (BPA) and NAG5-3248 (JEB) to the University of Toledo.

\section{References}

Bjorkman, J.E., Cassinelli, J.P. 1993, ApJ 409, 429

Cohen, D.H., Cassinelli, J.P., MacFarlane, J.J. 1997, ApJ 487, 867

MacFarlane, J.J., Cohen, D.H., Wang, P. 1994, ApJ 437, 351

MacFarlane, J.J., et al. 1993, ApJ 419, 813

Prinja, R.K. 1989, MNRAS 241, 721

Snow, T.P. 1981, ApJ 251, 139 